\title{
Origin of the dust emission from Tycho's SNR
}

\author{
D. Ishihara ${ }^{1}$, H. Kaneda ${ }^{1}$, A. Furuzawa ${ }^{1}$, H. Kunieda ${ }^{1}$, T. Suzuki ${ }^{2}$, B.-C. Koo ${ }^{3}$, H.-G. Lee ${ }^{4}$, J.-J. Lee ${ }^{5}$, and T. Onaka ${ }^{6}$ \\ 1 Department of Physics, Nagoya University, Furo-cho, Chikusa-ku, Nagoya, Aichi, 464-8602, Japan \\ e-mail: ishihara@u.phys.nagoya-u.ac.jp \\ ${ }^{2}$ Institute of Space and Astronautical Science, Japan Aerospace Exploration Agency, 3-1-1, Yoshinodai, Sagamihara, Kanagawa, \\ 252-5210, Japan \\ 3 Department of Physics and Astronomy, Seoul National University, 599 Gwanak-ro, Gwanak-gu, Seoul 151-742, Korea \\ 4 Department of Astronomy and Astrophysics, University of Toronto, Toronto, ON M5S 3H4, Canada \\ 5 Astronomy and Astrophysics Department, Pennsylvania State University, University Park, PA 16802, USA \\ 6 Department of Astronomy, Graduate School of Science, University of Tokyo, 7-3-1 Hongo, Bunkyo-ku, Tokyo, 113-0033, Japan
}

Received 2 June 2010 / Accepted 20 September 2010

\section{ABSTRACT}

\begin{abstract}
Aims. We investigate the spatial distribution of dust emission around Tycho's SNR to understand its origin. We distinguish the dust associated with the SNR from that of the surrounding ISM.

Methods. We performed mid- to far-infrared imaging observations of the remnant at wavelengths of 9, 15, 18, 24, 65, 90, 140, and $160 \mu \mathrm{m}$ using the Infrared Camera and the Far-Infrared Surveyor onboard AKARI. We compared the AKARI images with the Suzaku $\mathrm{X}$-ray image and the ${ }^{12} \mathrm{CO}$ image of Tycho's SNR.

Results. All the AKARI images except the 9,140, and $160 \mu \mathrm{m}$ band images show a shell-like emission structure with brightness peaks at the northeast (NE) and northwest (NW) boundaries, sharply outlining part of the X-ray shell. The 140 and $160 \mu \mathrm{m}$ bands are dominated by cold dust emission from the surrounding ISM near the NE boundary.

Conclusions. We conclude that the dust emission at the NE boundary comes from the ambient cloud interacting with the shock front, while the origin of the dust emission at the NW boundary is rather unclear because of the absence of prominent interstellar clouds near the corresponding region. We cannot rule out the possibility that the latter is mostly of an SN ejecta origin.
\end{abstract}

Key words. ISM: supernova remnants

\section{Introduction}

Tycho's supernova remnant (SNR; G120.1+01.4) is the remnant of a type Ia supernova (SN) explosion (Krause et al. 2008), which was observed by Tycho Brahe in 1572. Its distance is estimated to be $1.5-3.1 \mathrm{kpc}$ in several ways (Kamper \& van den Bergh 1978; Schwarz et al. 1995; Albinson et al. 1986; Strom 1988). Tycho's SNR has been widely studied in the X-ray and radio continua. The remnant shows an $8^{\prime}$ diameter limb-brightened shell, where the layer of the swept-up material is very thin (Warren et al. 2005). Inside the shell, metal line emissions in X-ray spectra are produced predominantly by SN ejecta (Bamba et al. 2005; Decourchelle et al. 2001). Furuzawa et al. (2009) revealed the Fe-emitting ejecta expanding at speeds of $2800-3350 \mathrm{~km} \mathrm{~s}^{-1}$ by the Suzaku observations.

In the synchrotron radio emission, Reynoso et al. (1997) reported the current anisotropic expansion of the shock front at speeds of 0 .' $^{\prime} 45 \mathrm{yr}^{-1}$ for the northwest (NW) and 0 .' $^{\prime} 15 \mathrm{yr}^{-1}$ for the northeast (NE) shock boundaries, based on the VLA observations over a $10 \mathrm{yr}$ interval. Lee et al. (2004) suggest a possible interaction of this remnant with a dense ambient cloud toward the $\mathrm{NE}$ direction based on the ${ }^{12} \mathrm{CO}$ observations. Ghavamian et al. (2000) proposed the excitation of the pre-shock medium around the NE boundary by high-energy particles and/or fast neutral precursor from the $\mathrm{H} \alpha$ observations.

In contrast, infrared (IR) observations are relatively scarce, although Tycho's SNR has been known as an IR emitter for a long time. IRAS determined the IR flux of the remnant
(Saken et al. 1992), while ISO resolved the dust emission that originated in collisional heating (Douvion et al. 2001) around the shock front of Tycho's SNR. The latter also indicate that a large amount of cold dust is not associated directly with the remnant. In light of the chemical evolution of the universe, type Ia SN plays an important role in providing a significant fraction of the Fe group elements in the ISM. However, the dust formation in type Ia SNR ejecta has never been observed to date (e.g. Blair et al. 2007), while it has been reported for type II SNRs (e.g. Douvion et al. 2001; Rho et al. 2008; Lee et al. 2009; Sibthorpe et al. 2010) since the first detection from SN 1987A (Lucy et al. 1989; Mosley et al. 1989; Wooden 1993).

In this Letter, we present the latest fine and wide-area midto far-IR AKARI images of Tycho's SNR. By comparing them with $\mathrm{X}$-ray and ${ }^{12} \mathrm{CO}$ images, we discuss the origin and physical state of the dust emission around the shell of Tycho's SNR.

\section{Observation and data}

We performed two pointed observations toward Tycho's SNR: one with the Infrared Camera (IRC; Onaka et al. 2007) and the other with the Far-Infrared Surveyor (FIS; Kawada et al. 2007). The AKARI mid-IR 15 and $24 \mu \mathrm{m}$ band imaging observations were made on 2007 February 1 with the IRC. The spatial resolution is $\sim 2$.' 5 for each image, where the pixel size is $\sim 22^{\prime \prime} 34 \times 22^{\prime \prime} 34$. The data were processed by using the standard IRC imaging data 

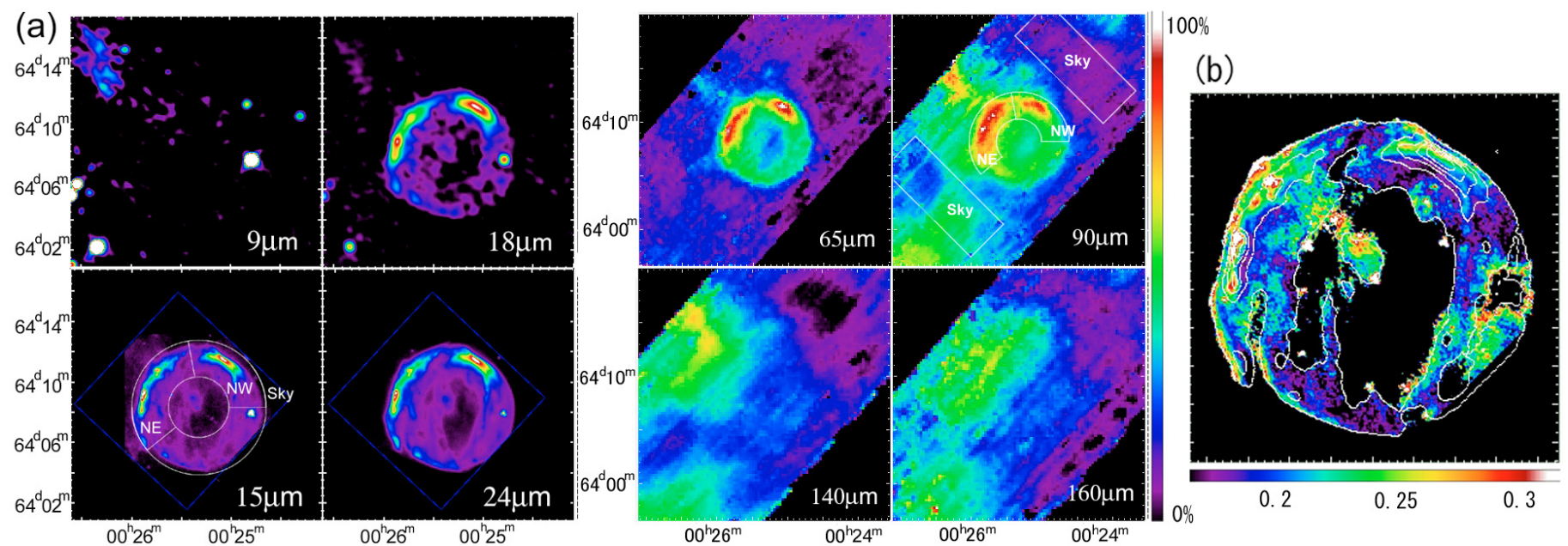

Fig. 1. a) AKARI 4-band mid-IR and 4-band far-IR images of Tycho's SNR drawn linearly in the color scales from zero to the maximum values of 5.3 $\mathrm{MJy} \mathrm{sr}^{-1}, 27 \mathrm{MJy} \mathrm{sr}^{-1}, 15 \mathrm{MJy} \mathrm{sr}^{-1}, 62 \mathrm{MJy} \mathrm{sr}^{-1}, 24 \mathrm{MJy} \mathrm{sr}^{-1}, 26 \mathrm{MJy} \mathrm{sr}^{-1}, 110 \mathrm{MJy} \mathrm{sr}^{-1}$, and $150 \mathrm{MJy} \mathrm{sr}^{-1}$ for the $9 \mu \mathrm{m}, 18 \mu \mathrm{m}, 15 \mu \mathrm{m}, 24 \mu \mathrm{m}$, $65 \mu \mathrm{m}, 90 \mu \mathrm{m}, 140 \mu \mathrm{m}$, and $160 \mu \mathrm{m}$ band images, respectively. The $9 \mu \mathrm{m}$ and $18 \mu \mathrm{m}$ images are created from the All-Sky Survey data, making the spatial resolution and sensitivity slightly worse than the $15 \mu \mathrm{m}$ and $24 \mu \mathrm{m}$ images from the pointed observation data. The FOVs in the pointed observations were shown in blue boxes in the $15 \mu \mathrm{m}$ and $24 \mu \mathrm{m}$ images. The definitions of the photometric apertures and sky regions for 9-24 $\mu \mathrm{m}$ bands and 65-160 $\mu \mathrm{m}$ bands in the $15 \mu \mathrm{m}$ and $90 \mu \mathrm{m}$ images, respectively. b) The ratio map of the $15 \mu \mathrm{m}$ to the $24 \mu \mathrm{m}$ band. The white contours indicate the $4 \%, 20 \%, 34 \%, 49 \%$, and 63\% levels of the peak brightness in the $24 \mu \mathrm{m}$ emission. The bright foreground star (GSC $04023-01147$ ) seen at 3.5 away from the center to the west in the panel a) is removed in the panel b) and Figs. 3 and 4.

reduction pipeline (version 20071017) ${ }^{1}$. The far-IR 65, 90, 140, and $160 \mu \mathrm{m}$ band images were taken on 2007 February 1 with the FIS in two round-trip slow scans (Kawada et al. 2007), where the scan speed was $15^{\prime \prime} \mathrm{s}^{-1}$. The spatial resolution is $30^{\prime \prime}$ for 65 and $90 \mu \mathrm{m}$ and $45^{\prime \prime}$ for 140 and $160 \mu \mathrm{m}$, and the bin size of each image is set to be $25^{\prime \prime}$. The data were processed with the FIS Slow-Scan Toolkit (version 20070914) ${ }^{1}$ and corrected for cosmic-ray effects (Suzuki et al. 2007).

The $9 \mu \mathrm{m}$ and $18 \mu \mathrm{m}$ band wide-area $\left(1^{\circ} \times 1^{\circ}\right)$ images around Tycho's SNR were created from the mid-IR All-Sky Survey data (Ishihara et al. 2006). The original pixel scale of the image is $99^{\prime} 36 \times 9$ '.36. The data were processed by the pipeline developed for the point source catalog (Ishihara et al. 2010), and additional custom procedures were applied in the same manner as described in Ishihara et al. (2007).

The 0.4-10 keV Suzaku/XIS X-ray image was taken from the DARTS ${ }^{2}$ archives at ISAS/JAXA. The ${ }^{12} \mathrm{CO}(1-0)$ images were taken from the archives of the Canadian Galactic Plane Survey (CGPS; Taylor et al. 2003), where we integrated the data using the two velocity ranges: one is from $-68 \mathrm{~km} \mathrm{~s}^{-1}$ to $-55 \mathrm{~km} \mathrm{~s}^{-1}$ and the other from $-63 \mathrm{~km} \mathrm{~s}^{-1}$ to $-60 \mathrm{~km} \mathrm{~s}^{-1}$. They are the likely maximal and minimal ranges for the clouds associated and interacting with the SNRs on the basis of the previous work (Lee et al. 2004).

\section{Results}

Figure 1 shows the AKARI multi-band images of Tycho's SNR, together with the ratio map of the $15 \mu \mathrm{m}$ to the $24 \mu \mathrm{m}$ band. The $15 \mu \mathrm{m}, 18 \mu \mathrm{m}$, and $24 \mu \mathrm{m}$ images clearly show a limb-brightened shell-like structure with several faint filaments. There are strong emissions at around the NW and NE boundaries. These bands contain important ionic line emissions such as [Ne II] at $12.8 \mu \mathrm{m}$ and [Ne III] at $15.6 \mu \mathrm{m}$, and pure rotational lines of molecular

\footnotetext{
${ }^{1}$ http://wWw.ir.isas.jaxa.jp/ASTRO-F/Observation/ DataReduction/

2 Data Archives and Transmission System (DARTS) at ISAS/JAXA http://www . darts.isas.ac.jp/astro/suzaku/
}

hydrogen such as $\mathrm{S}(1) J=3-1$ at $17.03 \mu \mathrm{m}$ and $\mathrm{S}(2) J=4-2$ at $12.28 \mu \mathrm{m}$. We estimated the contribution of the line emissions to the in-band fluxes by convolving the Spitzer/IRS spectra of typical SNRs (W44, W28, 3C391, IC443; Neufeld et al. 2007) with the spectral response curves of the IRC (Onaka et al. 2007). As a result, the contribution of the total line emissions is $36-52 \%$ for L15, 12-26\% for L18W, and 6-10\% for L24. Therefore, there may be non-negligible contributions from line emissions to the MIR intensities, but a major fraction of the intensities come from the continuum emission.

The $9 \mu \mathrm{m}$ emission is relatively faint and is not significantly detected from the shell structure of the remnant. The unusual faintness of the $9 \mu \mathrm{m}$ emission from the SNR is clearly recognized by making a comparison with the ISM cloud located at the NE corner in the $9 \mu \mathrm{m}$ and $18 \mu \mathrm{m}$ images. The $9 \mu \mathrm{m} / 18 \mu \mathrm{m}$ brightness ratios are $<0.003$ at the boundary and they are $\sim 3$ for the ISM cloud. The $9 \mu \mathrm{m}$ band includes the polycyclic aromatic hydrocarbon (PAH) features at 6.2, 7.7, 8.6, and $11.3 \mu \mathrm{m}$. Their faintness is compatible with the PAHs being destroyed effectively in SNRs (e.g. Tielens 2008).

The $65 \mu \mathrm{m}$ and $90 \mu \mathrm{m}$ band images also show the shelllike structure, while the $140 \mu \mathrm{m}$ and $160 \mu \mathrm{m}$ band images reveal the dominance of interstellar cold dust emission around the NE boundary; the latter does not appear to be associated with the remnant. The contribution of synchrotron emission to the far-IR fluxes is negligible. From the $20 \mathrm{~cm}$ radio flux of $10-40 \mathrm{mJy}$ with a spectral index of $-0.4 \sim-0.6$ for each of the NE and NW regions (Katz-Stone et al. 2000), we estimate the contribution of synchrotron emission to be $<1 \%$ of the observed far-IR fluxes.

We derived the flux densities of Tycho's SNR for the total, the NE, and the NW regions, separately, and definitions of the photometric apertures are shown in the $15 \mu \mathrm{m}$ and $90 \mu \mathrm{m}$ images in Fig. 1a. For each region, the resulting spectral energy distribution (SED) is then fitted by a two-temperature graybody model (Fig. 2). The temperatures and masses of the dust thus derived, as well as the flux densities, are summarized in Table 1. In the estimate of the dust mass, we assume a dust mass absorption coefficient of $28 \mathrm{~cm}^{2} \mathrm{~g}^{-1}$ at $90 \mu \mathrm{m}$ (Hildebrand 1983). 


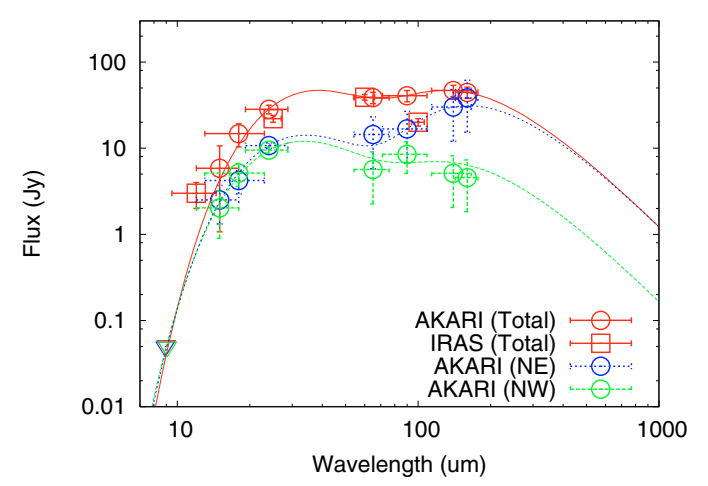

Fig. 2. SEDs for the total (red solid line), the NE (blue dashed), and the NW region (green dotted) of Tycho's SNR, fitted by a two-temperature graybody model. The AKARI and IRAS measurements are indicated by the open circles and the open squares, respectively.

Table 1. Infrared flux and its properties of the NW and NE regions of Tycho's SNR.

\begin{tabular}{cccc}
\hline \hline $\begin{array}{c}\text { Wavelength } \\
(\mu \mathrm{m})\end{array}$ & Total & Flux $(\mathrm{Jy})$ \\
\hline $8.61(4.10)$ & $<0.05$ & $<0.05$ & $\mathrm{NE}$ \\
$15.6(5.98)$ & $5.9 \pm 4.8$ & $2.0 \pm 1.1$ & $2.5 \pm 1.2$ \\
$18.4(9.97)$ & $14.8 \pm 4.4$ & $5.1 \pm 1.5$ & $4.2 \pm 1.3$ \\
$22.9(5.34)$ & $28.3 \pm 3.2$ & $9.5 \pm 0.8$ & $10.7 \pm 0.8$ \\
$65(21.7)$ & $38.6 \pm 7.7$ & $5.7 \pm 1.1$ & $14.4 \pm 2.9$ \\
$90(37.9)$ & $40.6 \pm 8.1$ & $8.5 \pm 1.7$ & $16.8 \pm 3.4$ \\
$140(52.4)$ & $46.7 \pm 14.0$ & $5.1 \pm 1.5$ & $30.0 \pm 9.0$ \\
$160(34.1)$ & $44.5 \pm 13.4$ & $4.6 \pm 1.4$ & $38 \pm 12$ \\
\hline \multicolumn{4}{c}{ Fitting results } \\
$T_{1}(K)$ & $95 \pm 2$ & $109 \pm 7$ & $107 \pm 4$ \\
$T_{2}(K)$ & $25 \pm 1$ & $25 \pm 10$ & $22 \pm 1$ \\
$M_{\text {warm dust }}\left(\times 10^{-4} M_{\odot}\right)$ & $10 \pm 3$ & $2.0 \pm 0.4$ & $2.0 \pm 0.6$ \\
$M_{\text {cold dust }}\left(M_{\odot}\right)$ & $0.3 \pm 0.1$ & $0.03 \pm 0.01$ & $0.3 \pm 0.1$ \\
\hline \multicolumn{4}{c}{}
\end{tabular}

Notes. ${ }^{(*)}$ The numbers in the parentheses are effective bandwidths (Onaka et al. 2007; Kawada et al. 2007).

As seen in Fig. 2, the AKARI measurements for the SED of the entire remnant show overall agreement with the previous ones (Saken et al. 1992) except at $100 \mu \mathrm{m}$, where IRAS gives a significantly lower value. We suspect that the IRAS flux is affected by the presence of the cold dust emission around the NE boundary in subtracting sky background. All the SEDs are reproduced with dust temperatures of $T_{1} \sim 100 \mathrm{~K}$ and $T_{2} \sim 20 \mathrm{~K}$, and the former can be interpreted by collisionally heated dust in the postshock plasma. The SED of the NE region, however, needs a large amount $\left(0.3 M_{\odot}\right)$ of cold $(\sim 20 \mathrm{~K})$ dust, which is likely attributed to the pre-existing ISM as already suggested by Douvion et al. (2001) and spatially resolved in AKARI far-IR images (Fig. 1a).

Figure $1 \mathrm{~b}$ shows the ratio map of the $15 \mu \mathrm{m}$ to the $24 \mu \mathrm{m}$ band. The ratios were calculated after subtracting background in each band, which was estimated by averaging the brightness of blank-sky areas surrounding the SNR. In this map, the ratios of 0.18 and 0.46 correspond to dust temperatures of $100 \mathrm{~K}$ and $136 \mathrm{~K}$, respectively, when we assume that these bands are dominated by thermal dust emission with the emissivity powerlaw index of 1.0. Thus these values are roughly consistent with the above warmer dust component. The figure shows that the dust temperature reaches a local maximum near the shock front, decreasing toward inner regions. As a whole, high-temperature

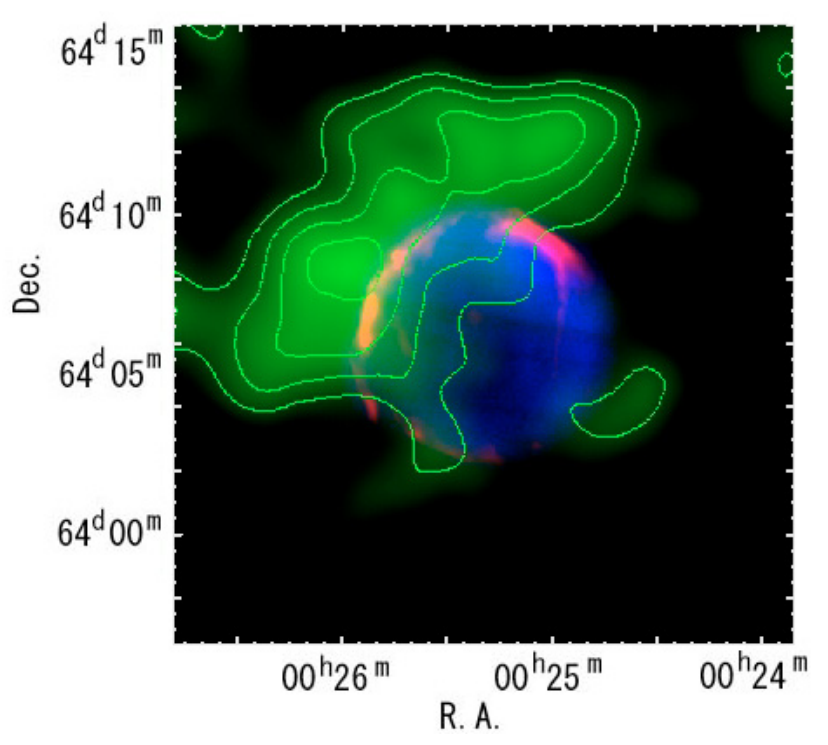

Fig. 3. Composite image of Tycho's SNR, consisting of AKARI $24 \mu \mathrm{m}$ band intensity in red, the CGPS ${ }^{12} \mathrm{CO}$ intensity integrated over the velocity range from -68 to $-55 \mathrm{~km} \mathrm{~s}^{-1}$ in green, and Suzaku $0.4-10 \mathrm{keV}$ $\mathrm{X}$-ray intensity in blue, The X-ray intensity is in the range of $2.6-13 \times$ $10^{-5}$ counts s${ }^{-1}$, and the $24 \mu \mathrm{m}$ intensity in the range of $4-40 \mathrm{MJy} \mathrm{sr}^{-1}$. The contours correspond to the brightness temperatures of $0.28,0.43$, 0.57 , and $0.71 \mathrm{~K}$ for the $\mathrm{CO}$ integrated intensity.

regions are distributed more isotropically around the shell than the dust emission itself, suggesting that the dust is shock-heated at the shell boundary. The systematic decrease in the ratio toward inner regions may indicate that smaller grains are mainly destroyed by sputtering.

\section{Discussion}

Figure 3 shows the composite images of Tycho's SNR, consisting of the AKARI mid-IR $(24 \mu \mathrm{m})$, the Suzaku X-ray $(0.4-10 \mathrm{keV})$, and the CGPS ${ }^{12} \mathrm{CO}$ images. In the figure, the $\mathrm{X}$-ray emission, which shows a circular outer shell, is surrounded by the ${ }^{12} \mathrm{CO}$ emission. They depict the past isotropic expansion and the current interaction with the dense medium at the NE boundary. Part of the X-ray shell is sharply outlined by the dust emission. We discuss the origins of these dust emissions below. First, we show that the dust cannot be totally destroyed by sputtering in the post-shock hot plasma. The dust have resided in the plasma for $\sim 50 \mathrm{yr}$ with the current shock speed and the thickness of the IR emission region, while the sputtering destruction timescale of the grains with a radius as small as $1 \mathrm{~nm}$ is estimated to be $150 \mathrm{yr}$ (Tielens et al. 1994) for the plasma temperature and density of $8.4 \times 10^{6} \mathrm{~K}$ and $10 \mathrm{~cm}^{-3}$ (Warren et al. 2005), respectively.

As described above, strong cold dust emission toward the $\mathrm{NE}$ direction is detected in the 140 and $160 \mu \mathrm{m}$ images (Fig. 1a). Indeed, the SED at the NE boundary exhibits the presence of a large amount of cold dust (Fig. 2). The distribution of the cold dust shows a spatial correspondence with the ${ }^{12} \mathrm{CO}(1-0)$ cloud surrounding the NE part of the remnant (Fig. 3), which are probably located close to the SNR from the ${ }^{12} \mathrm{CO}$ line velocities. The mid-IR dust emission comes from the outer edge of the cold dust and molecular cloud. Thus for the NE boundary, the midIR emission is very likely to originate in the shock-heated dust through interaction of the SNR with the ambient cloud. Unlike the NE boundary, the NW boundary region does not show the 


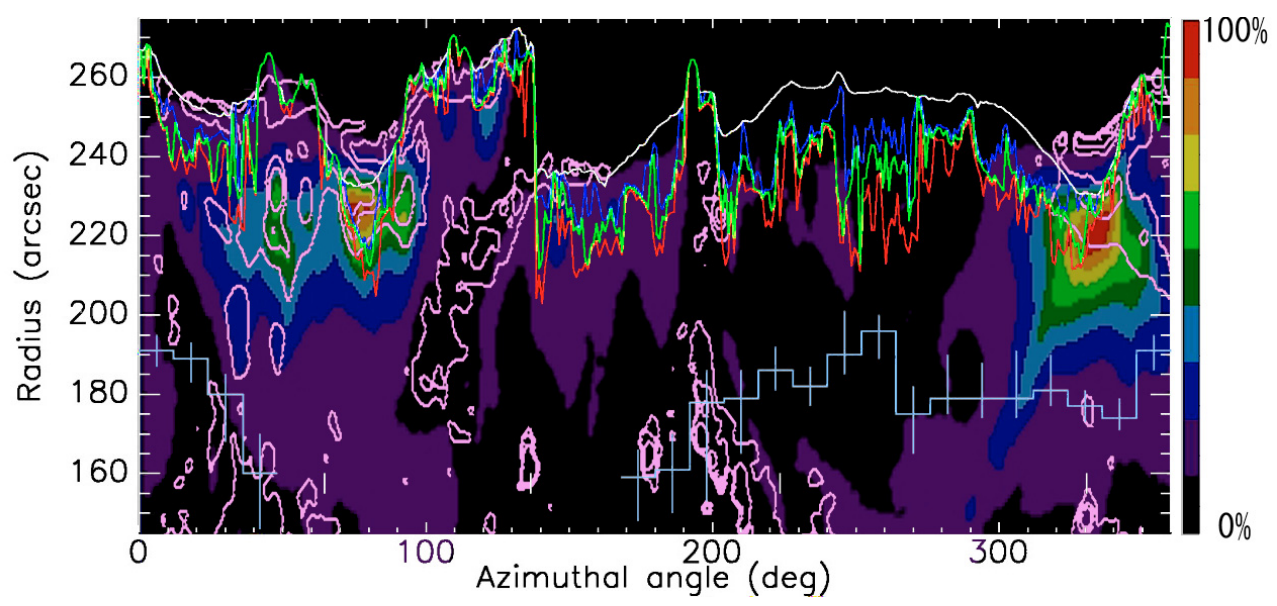

Fig. 4. AKARI $24 \mu \mathrm{m}$ intensity in the color, as well as the magenta contours of the $15 \mu \mathrm{m}$ to $24 \mu \mathrm{m}$ band ratio, are shown in a plane of the radius versus azimuthal angle. The color scale is linear with a maximum value of $62 \mathrm{MJy} \mathrm{sr}^{-1}$, while the contour levels correspond to the band ratios of $0.24,0.26$, and 0.28 . The center position is $\mathrm{RA}=00^{\mathrm{h}} 25^{\mathrm{m}} 19^{\mathrm{s}} .40$, Dec $=+64^{\circ} 08^{\prime} 13^{\prime \prime}$. 98 . The azimuthal angle is measured from the north through the east. For comparison, the positions of the blast wave (the top white curve), the contact discontinuity ( $2 \sigma:$ red, $3 \sigma:$ green, $4 \sigma: b l u e$ ), and the reverse shock (cyan) are shown together, which are taken from Warren et al. (2005).

clear presence of ISM clouds in the cold dust and ${ }^{12} \mathrm{CO}$ emissions. There are no HI clouds around the corresponding region (Reynoso et al. 1999). One possibility is that clouds, which were present there, have already been dispersed into ionized gas, and only dust remains. The highly anisotropic morphology, however, may not favor this scenario.

To make a more quantitative comparison between the NE and the NW boundary, we estimate the molecular gas masses using the same apertures as in Fig. 1a with the ${ }^{12} \mathrm{CO}$ X-factor of a typical Galactic value, $3 \times 10^{20} \mathrm{~cm}^{-2} \mathrm{~K}^{-1} \mathrm{~km}^{-1} \mathrm{~s}$. Integrating over the velocity range of -68 to $-55 \mathrm{~km} \mathrm{~s}^{-1}$, we derive the gas masses of 90 and $30 M_{\odot}$ in the NE and the NW region, respectively, while they are 20 and $2 M_{\odot}$ for the velocity range of -63 to $-60 \mathrm{~km} \mathrm{~s}^{-1}$. Thus, from Table 1, the gas-to-dust mass ratios are 70-300 in NE and 70-1000 in NW for the cold dust, while they are $(1-5) \times 10^{5}$ in NE and $(0.1-2) \times 10^{5}$ in NW for the warm dust. As for the cold dust, the ratios in NE and NW are similar to each other around a typical ISM value (100-200; Sodroski et al. 1994), although the NW ratio has a large uncertainty depending on the adopted velocity range. Therefore the cold dust is likely to be of a pre-existing ISM origin. However for the warm dust, which is collisionally heated by the SNR, the gas-to-dust mass ratio in NW is systematically less than in the NE. If we adopt more restricted velocity range, which is more appropriate to the interacting part of the cloud, the difference is as much as a factor of 10 . Therefore we conclude that the NW region is relatively rich in warm dust.

Figure 4 shows the distribution of the dust emission compared to the positions of the blast wave, the contact discontinuity (CD), and the reverse shock, all of which are plotted in a plane of the radius versus azimuthal angle. The NE bright spot at the angle of $\sim 80^{\circ}$ is located between the forward shock and the $\mathrm{CD}$, which is compatible with the picture of the NE dust mostly having an interstellar origin (i.e. swept-up materials). A local maximum of the dust temperature coincides well with the brightness peak. In contrast, the NW bright spot $\left(\sim 330^{\circ}\right)$ is located between the reverse shock and the $\mathrm{CD}$. The dust temperature does not peak at the NW bright spot (see also Fig. 1b). Thus the situation is considerably different between the NE and NW bright spots. Judging from these, combined with the filamentary structures extended toward inner regions, a majority of the NW dust could have an SN ejecta origin.
It should be noted that the projection effect can explain the difference because the swept-up layer of this remnant is very thin; however Warren et al. (2005) showed Si-rich X-ray spectra from the NW region where the mid-IR dust emission is bright, suggesting that the emitting matter comes from ejecta rather than from ISM. With AKARI, Lee et al. (2009) detected dust emission from ejecta of the type II SNR, G292.0+1.8, which has the $15 \mu \mathrm{m}$ to $24 \mu \mathrm{m}$ band ratio of $>0.5$. If the NW dust emission also comes from ejecta, the band ratio is significantly lower than the above ratio, which may possibly be attributed to the difference in chemical composition of dust between types Ia and II SNRs.

\section{Summary}

We have presented the latest fine and wide-area mid- to far-IR AKARI images of Tycho's SNR, which are compared with the $\mathrm{X}$-ray and ${ }^{12} \mathrm{CO}$ images. They show a shell-like emission structure with bright peaks at the NE and NW boundaries, sharply outlining part of the X-ray image. Most of the mid-IR dust emission comes from the dust shock-heated at the shock front. A significant fraction of the far-IR and the PAH emission comes from the ISM clouds near the NE shock boundary, which further reveals a large-scale jet-like structure in front of the NE boundary with a spatial correspondence in the ${ }^{12} \mathrm{CO}$ emission. We conclude that the mid-IR dust emission at the NE boundary comes from the ISM interacting with the shock front, judging from the spatial correlation among the mid-IR dust, the cold dust, and the molecular cloud. The origin of the dust emission at the NW boundary is rather unclear due to the absence of prominent interstellar clouds near the corresponding region. We estimated gas-to-dust mass ratios at the NE and the NW boundary to reveal that the NW region is very rich in warm dust. We find that a large fraction of the NW dust emission comes from the region between the reverse shock and the $\mathrm{CD}$, assuming there is no projection effect. We therefore cannot rule out the possibility that a major fraction of the dust emission at the NW boundary is of an $\mathrm{SN}$ ejecta origin.

Acknowledgements. This research is based on observations with AKARI, a JAXA project with the participation of ESA. This study was initiated by preliminary studies done by high school students who visited Nagoya University for a one-week internship. We have made use of the NASA/IPAC Infrared 
Science Archive, which is operated by the Jet Propulsion Laboratory, California Institute of Technology, under contract with the National Aeronautics and Space Administration, and archival data from the Canadian Galactic Plane Survey (CGPS), a Canadian project with international partners, supported by the Natural Sciences and Engineering Research Council. We also thank A. Kawamura for providing precious suggestions for analyzing of the molecular clouds. This work was supported by the the Nagoya University Global COE Program, "Quest for Fundamental Principles in the Universe (QFPU)" from JSPS and MEXT of Japan. We also express many thanks to the anonymous referee for a careful reading and constructive comments.

\section{References}

Albinson, J. S., Tuffs, R. J., Swinbank, E., \& Gull, S. F. 1986, MNRAS, 219, 427 Bamba A., Yamazaki, R., Yoshida, T., Terasawa, T., \& Koyama, K. 2005, ApJ, 621, 793

Blair, W. P., Ghavamian, P., Long, K. S., et al. 2007, ApJ, 662, 998

Decourchelle, A., Sauvageot, J. L., Audard, M., et al. 2001, A\&A, 365, L218

Douvion, T., Lagage, P. O., Cesarsky, C. J., \& Dwek, E. 2001, A\&A, 373, 281

Furuzawa, A., Ueno, D., Hayato, A., et al. 2009, ApJ, 693, L61

Ghavamian, P., Raymond, J., Hartigan, P., \& Blair, W. 2000, ApJ, 535, 266

Ishihara, D., Wada, T., Onaka, T., et al. 2006, PASP, 118, 324

Ishihara, D., Onaka, T., Kaneda, H., et al. 2007, PASJ, 59, S443

Ishihara, D., Onaka, T., Kataza, H., et al. 2010, A\&A, 514, 1

Hildebrand, R. H. 1983, QJRAS, 24, 267

Kamper, K. W., \& van den Bergh, S. 1978, ApJ, 224, 851

Kawada, M., Baba, H., Barthel, P. D., et al. 2007, PASJ, 59, S389
Katz-Stone, D. M., Kassim, N. E., Lazio, T. J. W., \& O’Donnell, R. 2000, ApJ, 529,453

Krause, O., Tanaka, M., Usuda, T., et al. 2008, Nature, 456, 617

Lee, H. G., Koo, B. C., Moon, D. S., et al. 2009, ApJ, 706, 441

Lee, J. J., Koo, B. C., \& Tatematsu, K. 2004, ApJ, 605, L113

Lucy, L. B., Danziger, I. J., Gouiffes, C., \& Bouchet, P. 1989, LNP, 350, 164

Moseley, S. H., Dwek, E., Glaccum, W., Graham, J. R., \& Loewenstein, R. F. 1989, Nature, 340, 697

Neufeld, D. A., Hollenbach, D. J., Kaufman, M.J., et al. 2007, ApJ, 664, 890

Onaka, T., Matsuhara, H., Wada, T., et al. 2007, PASJ, 59, S401

Reynoso, E. M., Moffett, D. A., Goss, W. M., et al. 1997, ApJ, 491, 816

Reynoso, E. M., Velázquez, P. F., Dubner, G. M., \& Goss, W. M. 1999, ApJ, 117, 1827

Rho, J., Kozasa, T., Reach, W. R., et al. 2008, ApJ, 673, 271

Saken, J. M., Fesen, R. A., \& Shull, J. M. 1992, ApJS, 81, 715

Schwarz, U. J., Goss, W. M., Kalberla, P. M., \& Benaglia, P. 1995, A\&A, 299, 193

Sibthorpe, B., Ade, P. A. R., Bock, J. J., et al. 2010, ApJ, 719, 1553

Sodroski, T. J., Bennett, C., Boggess, N., et al. 1994, ApJ, 428, 638

Strom, R. G. 1988, MNRAS, 230, 331

Suzuki, T., Kaneda, H., Nakagawa, T., et al. 2007, PASJ, 59, 473

Taylor, A. R., Gibson, S. J., Peracaula, M., et al. 2003, AJ, 125, 3145

Tielens, A. G. G. M. 2008, ARA\&A, 46, 289

Tielens, A. G. G. M., McKee, C. F., Seab, C. G., \& Hollenbach, D. J. 1994, ApJ, 431,321

Warren, J. S., Hughes, J. P., Badenes, C., et al. 2005, ApJ, 634, 376

240, 7

Wooden, D. H., Rank, D. M., Bregman, J. D., et al. 1993, ApJS, 88, 477 